\title{
SQL ALGORITHM FOR SOLVING MARKOV MODELS BY GRAPH METHOD
}

A simple graph algorithm for finding stabilized probabilities of the finite Markov models implemented in SQL is presented. The algorithm generates systematically all oriented spanning trees of a transition graph. The method is demonstrated on the computation of probabilities in the $M M P P_{2} / M / 1 /$ K queue.

Keywords: Markov models, queue, graph algorithm, SQL algorithm

\section{Introduction}

The original graph study of steady-state distribution for stabile Markov process with finite state sets is presented by Markl [1]. Stabilized probabilities $\pi$ of the stable Markov process associated with a weighted digraph (called transition graph) $G=(S, E, c)$ are given by formulas

$$
\pi i=\frac{B_{i}}{\sum_{j \in S} B_{j}} i \in S,
$$

where $B_{i}, i \in S$ is the sum of weights of all the spanning trees of $G$ that have their roots in vertex $i$.

For processes with many states and many possible transitions between them is not easy to find all spanning trees. We apply a simple algorithm that systematically generates all spanning trees with given roots in SQL.

\section{Basic definitions}

We start with giving the some definitions from graph theory that will be used in this paper. A (simple) digraph $G=(V, E)$ consists of a finite set $V$ of vertices and set $E$ of edges - ordered pairs of distinct vertices; that is, each edge $(u, v)$ is directed from tail $u$ to head $v$. A (directed) $u-v$ path from vertex $u$ to vertex $v$ is such a sequence $u=v_{0},\left(v_{0}, v_{1}\right), v_{1},\left(v_{1}, v_{2}\right), v_{2}, \ldots,\left(v_{k-1}, v_{k}\right), v_{k}=v$ that $v_{i} \in V$ for $i=0 . \ldots, k$ and $v_{i} \neq v_{j}$ for $0<i<j<k$, and that $\left(v_{i-1}, v_{i}\right) \in E$ for $i=1, \ldots, k$. If $u=v$ then $u-v$ path is called a cycle. A digraph in which each pair of vertices lies on a common cycle is called strongly connected. A digraph in which for each pair $\{u, v\} \in V$ exists $u-v$ path or $v-u$ path is called connected. The component of a digraph is maximal connected subgraph of a digraph.

A digraph that has no cycle is called a directed acyclic graph (DAG). A rooted tree is a DAG in which one vertex, the root, is distinguished and in which all edges are implicitly directed to the root. (Note that in the standard definition [7] of the rooted tree the orientation of the edges is away from the root.) The weight of the (edge) weighted rooted tree $T=\left(V, E_{T}, w\right)$ we mean number

$$
w(T)=\prod_{h \in E_{T}} w(h)
$$

A spanning rooted tree $(T, r)$ with root $r$, shortly spanning $r$-tree, of a strongly connected digraph $G$ is a rooted tree $T$ that is a subgraph of $G$ and that contains every vertex of $G$. The weight of the a spanning $r$-tree $(T, r)$ of a (edge) weighted digraph $G=$ $=(V, E, w)$ is weight $w(T)$ of the rooted tree $T=\left(V, E_{T}\right)$ given by (2).

\section{SQL algorithm}

The strongly connected digraph $G=(V, E)$ is given. We may assume without loss of generality that $V=\{1,2, \ldots, n\}$ and root $r=1$. The cases where $r \neq 1$ can be transformed to the case $r=1$ by renumbering of vertices $V$. Let the set $E$ be represented by a table Edge with two columns:

- Edge.u is head of a directed edge $(u, v)$

- Edge.v is tail of a directed edge $(u, v)$

and a table Solution with two columns of $\operatorname{array}[1, \ldots, n]$ :

- Solution.tree is the subtree - subgraf of spanning 1-tree

- Solution.comp is the components of tree.Solution

In Figure 1 we have the example of the spanning 1-tree $T=$ $=\left(V, H_{T}\right)$ where $V=\{1,2,3,4,5\}$ and $H_{T}=\{(2,4),(3,5),(4,1)$, $(5,4)\}$ are represented by array tree $=[0,4,5,14]$. In Figure 2

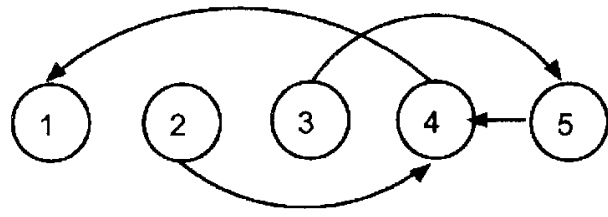

Fig. 1 The spanning 1-tree as array [0, 4, 5, 1, 4]

\footnotetext{
* Štefan Peško

Department of Mathematical Methods, Faculty of Management Science and Informatics, University of Žilina, Slovakia,

E-mail: pesko@frcatel.fri.utc.sk
} 
we have the example of the subtree of the spanning 1-tree which is represented by array tree $=[0,4,0,1,4]$ and has two components $\left.T_{1}=\{1,2,4,5\},\{(2,4),(4,1),(5,4)\}\right)$ and $T_{2}=(\{3\}, \varnothing)$ which are represented by array comp $=[1,1,3,1,1]$.

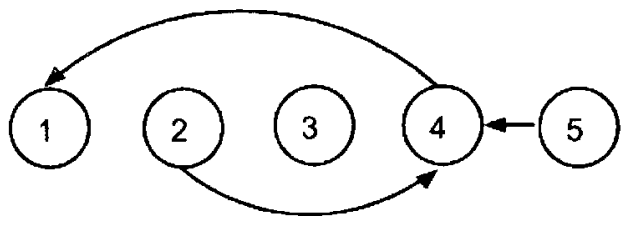

Fig. 2 The subtree of the 1-tree with two components

We can now describe the algorithm which generates all spanning 1-trees:

procedure SQL1Trees (Edge )

for $i=1$ to $n$ do (*Initialization $\left.{ }^{*}\right)$

Solution.tree $[i]=0$, Solution.comp $[i]=i$

for $k=1$ to $n-1$ do

SELECT DISTINCT ( Subgraphs of spanning 1-trees *)

SetValue (Solution.tree, Edge.u, Edge.v) AS tree

SetValue (Solution.comp, Edge.u, 1) AS comp

FROM Solution, Edge

WHERE

Solution.comp [Edge.u] $\neq 1$ AND Solution.comp

[Edge.v] $=1$

GROUP BY tree, comp

TO FILE Solution

Edges of the transition graph $G$

Tab. 1

\begin{tabular}{|c|c|c|c|c|c|c|c|c|c|c|c|c|c|}
\hline $\mathrm{u}$ & 1 & 1 & 2 & 3 & 3 & 3 & 4 & 4 & 4 & 5 & 5 & 6 & 6 \\
\hline $\mathrm{v}$ & 2 & 3 & 1 & 1 & 4 & 5 & 2 & 3 & 6 & 3 & 6 & 4 & 5 \\
\hline
\end{tabular}

Before we start the main SQL-algorithm, we calculate the values of null subtree $(\mathrm{V}, \varnothing)$ with $n$ components. One new edge $(u$, $v$ ) appends in the $k$-step, if it is possible else subtree is deleted. So the subtrees $\left(V, H_{k}\right)$ where $\left|H_{k}\right|=k$ are generated step by step. After the last step we have all spanning 1-trees.

A new subtree and its components are stored in an array tree and comp which are updated by the function:

$$
\begin{aligned}
& \text { function } x=\operatorname{SetValue}(x, i, j) \\
& x[i]=j
\end{aligned}
$$

Note that after the last step are comp $=[1,1,1, \ldots, 1]$ because the spanning 1-trees are connected digraphs.

\section{Example of $\mathrm{MMPP}_{2} / \mathrm{M} / 1 / \mathrm{K}$ queue}

We consider a queueing system studied by Peško [2] with twostate Markov modulated Poisson process $M M P P_{2}$, a single exponential distributed server and a finite waiting room. The problem of

switch design and admission control in a high speed network is modeled in [6] as $M M P P_{2} / G I / 1 / \infty$ queue. The transition graph for the $M M P P_{2} / M / 1 / K$ queue is a weighted digraph

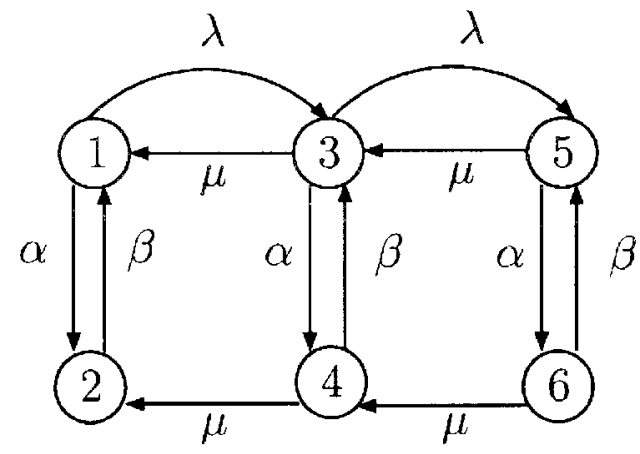

Fig. 3 Transition graph $G$ of $M M P P_{2} / M / 1 / 3$ queue

$G=(S, E, c)$. In Figure 3 we have the small example of the $M M P P_{2} / M / 1 / 3$ queue with maximal 2 customers in waiting room where $\alpha$ and $\beta$ are ON and OFF rate of source, $\lambda$ is the arrival rate from $\mathrm{ON}$ source and $\mu$ the service rate.

We have a table Edge in the Table 1. The costs of the edges of the transition graph are omitted. Note that the table Edge is shown horizontally instead of a usual vertical layout. The initialization table Solution is in the first row of the Table 2. After $k^{\text {th }}$ step $(k=$ $=1,2,3,4)$ we have a new Solution in Table 2 with added column of step marked $k$. All spanning 1-trees are generated in the last Solution table 3 and described in figure 4 . And so $B_{1}$ - the sum of weights of all the spanning 1-trees is

$\alpha \prod_{0}^{\mu} \stackrel{\mu}{\Perp}$

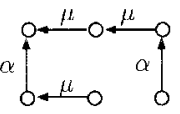

$[0,1,1,2,3,5]$
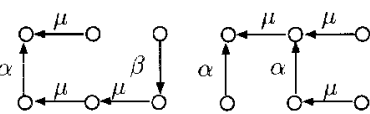

$[0,1,1,2,3,4]$

$[0,1,1,2,6,4]$

$[0,1,1,3,3,4]$
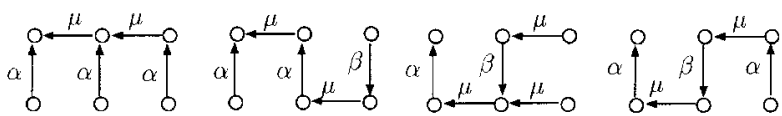

$[0,1,1,3,3,5]$

$[0,1,1,3,6,4]$

$[0,1,4,2,3,4]$

$[0,1,4,2,3,5]$

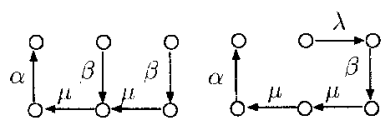

$[0,1,4,2,6,4] \quad[0,1,5,2,6,4]$

Fig. 4 All spanning 1-trees of $G$

$$
\begin{aligned}
B_{1} & =\alpha \mu^{4}+2 \alpha^{2} \mu^{3}+\alpha^{3} \mu^{2}+2 \alpha \beta \mu^{3}+ \\
& +2 \alpha^{2} \beta \mu^{2}+\alpha \beta^{2} \mu^{2}+\lambda \alpha \beta \mu^{2} .
\end{aligned}
$$

The sum of weights of all the spanning $r$-trees $B_{r}$ for $r=2, \ldots$, 6 can be computed by renumbering set of vertices $S$. 
Tab. 2

\begin{tabular}{|c|c|c|}
\hline tree & comp & $\mathrm{k}$ \\
\hline$[0,0,0,0,0,0]$ & {$[1,2,3,4,5,6]$} & - \\
\hline$[0,1,0,0,0,0]$ & {$[1,1,3,4,5,6]$} & 1 \\
{$[0,0,1,0,0,0]$} & {$[1,2,1,4,5,6]$} & 1 \\
\hline$[0,1,1,0,0,0]$ & {$[1,1,1,4,5,6]$} & 2 \\
{$[0,1,0,2,0,0]$} & {$[1,1,3,1,5,6]$} & 2 \\
{$[0,0,1,3,0,0]$} & {$[1,2,1,1,5,6]$} & 2 \\
{$[0,0,1,0,3,0]$} & {$[1,2,1,4,1,6]$} & 2 \\
\hline$[0,1,1,2,0,0]$ & {$[1,1,1,1,5,6]$} & 3 \\
{$[0,1,1,3,0,0]$} & {$[1,1,1,1,5,6]$} & 3 \\
{$[0,1,1,0,3,0]$} & {$[1,1,1,4,1,6]$} & 3 \\
{$[0,1,4,2,0,0]$} & {$[1,1,1,1,5,6]$} & 3 \\
{$[0,1,0,2,0,4]$} & {$[1,1,3,1,5,1]$} & 3 \\
{$[0,0,1,3,3,0]$} & {$[1,2,1,1,1,6]$} & 3 \\
{$[0,0,1,3,0,4]$} & {$[1,2,1,1,5,1]$} & 3 \\
{$[0,0,1,0,3,5]$} & {$[1,2,1,4,1,1]$} & 3 \\
\hline$[0,1,1,2,3,0]$ & {$[1,1,1,1,1,6]$} & 4 \\
{$[0,1,1,2,0,4]$} & {$[1,1,1,1,5,1]$} & 4 \\
{$[0,1,1,3,3,0]$} & {$[1,1,1,1,6]$} & 4 \\
{$[0,1,1,3,0,4]$} & {$[1,1,1,1,5,1]$} & 4 \\
{$[0,1,1,0,3,5]$} & {$[1,1,1,4,1,1]$} & 4 \\
{$[0,1,4,2,3,0]$} & {$[1,1,1,1,1,6]$} & 4 \\
{$[0,1,4,2,0,4]$} & {$[1,1,1,1,5,1]$} & 4 \\
{$[0,1,0,2,6,4]$} & {$[1,1,3,1,1,1]$} & 4 \\
{$[0,0,1,3,3,4]$} & {$[1,2,1,1,1,1]$} & 4 \\
{$[0,0,1,3,3,5]$} & {$[1,2,1,1,1,1]$} & 4 \\
{$[0,0,1,3,6,4]$} & {$[1,2,1,1,1,1]$} & 4 \\
\hline
\end{tabular}

Solution after last $5^{\text {th }}$ step

Tab. 3

\begin{tabular}{|c|c|}
\hline tree & comp \\
\hline$[0,1,1,2,3,4]$ & {$[1,1,1,1,1,1]$} \\
{$[0,1,1,2,3,5]$} & {$[1,1,1,1,1,1]$} \\
{$[0,1,1,2,6,4]$} & {$[1,1,1,1,1,1]$} \\
{$[0,1,1,3,3,4]$} & {$[1,1,1,1,1,1]$} \\
{$[0,1,1,3,3,5]$} & {$[1,1,1,1,1,1]$} \\
{$[0,1,1,3,6,4]$} & {$[1,1,1,1,1,1]$} \\
{$[0,1,4,2,3,4]$} & {$[1,1,1,1,1,1]$} \\
{$[0,1,4,2,3,5]$} & {$[1,1,1,1,1,1]$} \\
{$[0,1,4,2,6,4]$} & {$[1,1,1,1,1,1]$} \\
{$[0,1,5,2,6,4]$} & {$[1,1,1,1,1,1]$} \\
\hline
\end{tabular}

The research of the autor is supported by the Slovak Scientific Grant Agency under grant No. 1/0490/03.

\section{References:}

[1] MARKL, J.: A Graph Method for Markov Models Solving, Acta Mathematica et Informatica Universitatis Ostraviensis, Vol. 1, 1993, pp. $75-82$

[2] PEŠKO, Š.: Erlang ON/OFF Moduled Queueing Systems, Proceedings of the 20'th International Conference, Mathematical Methods in Economics 2002, Ostrava, ISBN 80-248-0153-1, 2002, pp. 209-213

[3] ROSS, S. M.: Stochastic Processes, John Wiley \& Sons, Inc., 1983, ISBN 0-471-09942-2

[4] KAUKIČ, M.: Open Source Software in Mathematical Education, 1. International conference, Aplimat, Bratislava 2002, pp. 233-238

[5] REBO, J., BARTL, O.: Condition of Stability for Tandem Queues with Blocking and Exponential and Erlangian Service Time Distribution, Studies of the Faculty of Management Science and Informatics, Vol. 8, Žilina, 1999, pp. 67-74

[6] JEAN-MARIA, A., LIU, Z., NAIN, P., TOWSLEY, D.: Computational Aspects of the Workload Distribution in the MMPP/GI/1 Queue, IEEE Journal on Selected Areas in Communications, Vol.16, No.5, 1998, pp.640-652

[7] PALÚCH, S.: Graph Theory (Teória grafov), Žilinská univerzita, EDIS, 2001, ISBN 80-7100-874-5

[8] MATIAŠKO, K.: Database Systems (Databázové systémy), Žilinská univerzita, EDIS, 2002, ISBN 80-7100-968-7. 